\title{
IDH1 NM_005896.3:c.395_396delGTinsAC
}

National Cancer Institute

\section{Source}

National Cancer Institute. IDH1 NM 005896.3:c.395 396delGTinsAC. NCI Thesaurus.

Code C128900.

A complex substitution where the nucleotide sequence at positions 395 and 396 of the coding sequence of the IDH1 gene has changed from thymine-guanine to adeninecytosine. 